\title{
Investigating Performance of XML Web Services in Real-Time Business Systems
}

\author{
Alaa M. Riad', Ahmed E. Hassan², Qusay F. Hassan ${ }^{3}$ \\ ${ }^{1}$ Head of Information Systems Department, Mansoura University, Egypt \\ ${ }^{2}$ Teacher of Electrical Engineering in Faculty of Engineering, Mansoura University, Egypt \\ ${ }^{3} \mathrm{PhD}$ Researcher in Faculty of Computers and Information Systems, Mansoura University, Egypt
}

\begin{abstract}
Service-Oriented Architecture (SOA) is being one of the widely accepted methodologies in software market for building and integrating different kinds of software systems. This acceptance comes from the extreme benefits that it offers to their adopters including agility, dynamicity, and loose-coupling. These benefits are usually missed in traditional software terminologies and practices. XML Web Services is the most used technology for applying SOA because it is easy to use and it allows high interoperability between different systems due to its dependency on standards that are widely accepted and supported by almost all large software vendors. However, XML Web Services suffer from a number of drawbacks such as low performance, bad utilization for hardware resources, and high network latency. These pitfalls may prevent some adopters from utilizing SOA in large and complex systems. So, these issues should be first addressed and resolved before leveraging it into real-time systems. This paper presents an experimental evaluation for the performance of XML Web Services in real-time business systems. Furthermore, it offers some tactics and strategies that might be used to enhance the overall performance of XML Web Services.
\end{abstract}

Keywords: SOA; XML web services; Problem root causes; Systems integration; Performance evaluation; Optimization tactics and strategies

\section{Introduction}

Over the last few years, SOA has gained momentum from almost all players in software market for building and integrating systems, especially complex ones that demand continuous changes to meet market ever-changing requirements (Papazoglou, 2003; Channabasavaiah, 2003).

Technically, many technologies could be used for realizing and implementing service-oriented systems including message queuing, remote procedure calls (RPCs), Common Object Request Broker (CORBA), and Common Object Model (COM) (Krafzig et al., 2005). However, XML Web Services is the most used technology for realizing SOA due to number of factors including (Curbera et al., 2002):

- Ease-of-use: Using XML Web Services does not require deep technical knowledge, as it is very easy to learn and use, especially if compared with other tools like CORBA and COM.

- Support: Because it has appeared as a result of cooperation between large software vendors such as Microsoft, IBM,
BEA, and Sun Microsystems, it is supported in almost all software tools, frameworks, and programming languages.

- Modularity: It is modular by nature, so, it is easy to encapsulate logic in terms of modules that could be deployed and used separately according to business needs.

- Compose-ability: It is very easy to aggregate a number of XML Web Services to construct a new one that covers more complex needs.

- Low costs: It is much cheaper than traditional and proprietary technologies.

- Commonality with SOA model: The architectural model of XML Web Services is almost identical to the basic model of SOA, as they both have service provider, service consumer, service registry, service contract, and service itself.

Unfortunately, the aforementioned advantages of XML Web Services were not enough to enable SOA adopters to use it effectively in real-time business systems, as it still suffers from number of problems such as low performance, bad utilization for hardware resources, and high network latency. These issues must be first addressed and resolved to allow optimum utilization for SOA.

This paper examines the performance of XML Web Services in building and integrating real-time business systems taking a hypothetical scenario for banking solutions as an example to these systems in order to compare found results with those of using other traditional methods. Additionally, it discusses root causes of found problems in order to give some tactics and strategies that could be leveraged to make better use of XML Web Services.

\section{Root Causes}

Many studies have discussed the performance of XML Web Services from different perspectives, and they all concluded that poor performance goes back to a number of reasons including:

- XML data format: The technology of XML Web Services depends on XML (Extensible Markup Language) for repre-

\footnotetext{
*Corresponding author: Qusay F. Hassan, PhD Researcher in Faculty of Computers and Information Systems, Mansoura University, Egypt, E-mail: qusayfadhel@yahoo.com
}

Received September 29, 2009; Accepted October 24, 2009; Published October 29, 2009

Citation: Riad AM, Hassan AE, Hassan QF (2009) Investigating Performance of XML Web Services in Real-Time Business Systems. J Comput Sci Syst Biol 2: 266-271. doi:10.4172/jcsb.1000041

Copyright: ( 2009 Riad AM, et al. This is an open-access article distributed under the terms of the Creative Commons Attribution License, which permits unrestricted use, distribution, and reproduction in any medium, provided the original author and source are credited.

J Comput Sci Syst Biol

Volume 2(5): 266-271 (2009) - 266

ISSN:0974-7230 JCSB, an open access journal 


\section{Journal of Computer Science \& Systems Biology - Open Access}

\section{JCSB/Vol.2 September-October 2009}

senting data being transmitted between different systems and nodes. As known, XML is a tag-based language rich with different capabilities that allow easy and powerful integration between different systems. For example, it offers different mechanisms for validating, querying, and transforming data (XML, 2004). These capabilities made XML the preferred choice of software vendors who look for interoperability and loose-coupling between integrated systems. However, this richness causes data files to be bloated with long named tags, complex data structures, and big amounts of plain-text data that make their generation and processing very complex, heavy, and slow.

- Encoders/decoders: XML Web Services use encoders to transform data into a sequence of bytes before transmission, and on the other side, they use decoders to return transmitted data to its original form. ASCII was one of the most used encoding formats in legacy systems. Depending on ASCII format in XML Web Services is very expensive and slow especially for numerical values and floating points (Chiu et al., 2002).

- Parsing techniques: XML being transmitted between different nodes must be parsed and validates before any further processing. Using inefficient parsing techniques may require much hardware resources (including RAM and processing cycles) and long times to complete required tasks.

- Serialization/de-serialization: Serialization (marshaling) is the process that converts the state of objects in a form that can be transmitted over network media (such as wires and $\mathrm{Wi}-\mathrm{Fi}$ ) between different nodes. Conversely, de-serialization (de-marshaling) process is responsible for bringing the state information to original formats. Efficient serialization techniques must be very fast and generate serialized data in compact formats. Depending on bad serialization techniques may generate very large data outputs that might clog network during transmission process. As mentioned, XML Web Services serialize data using XML format, and because XML is text-based format, then generated messages are always very large in size if compared to original data (before serialization) (Chiu et al., 2002; Bustamante et al., 2000).

- Transport protocol: XML Web Services use Simple Object Access Protocol (SOAP) as a lightweight communication framework that is based on XML. Although SOAP messages can use any transport protocol to send requests and receive responses, it uses HTTP as a default transport protocol. HTTP is a request-response protocol that supports only synchronous interaction between clients and servers, and this makes it ill-suited for message-based communications that require asynchronous interactions (Fielding et al., 1999).

- Network infrastructure: There is no question that, network is one of the most important factors for the success of any client/server implementations, and thus, depending on weak and slow networks can lead to unreliable, inefficient, and intermittent interactions between clients and servers. Furthermore, slow networks may lead to less utilization for available processing power because CPUs will wait longer times until data arrives to be processed.

- Extra elements in software stack: In service-oriented sys- tems, XML Web Services are defined in a separate layer that accepts clients' requests to be formatted before sending them to underlying components. As known, the more layers and processing logic defined in any software architecture, the slower performance of overall system. This is due to extra processing that is needed to reach final element in software stack including discovery, reflection, initialization, instantiating, and invocations of needed components and objects as well as transformations for incoming requests and out-coming data formats.

\section{Technical Scenario}

Integrating different systems together is a very common scenario in software field, and leveraging SOA for building and integrating different business systems like banking solutions offers many advantages over other traditional integration methods including simplicity, dynamicity, agility, and loose-coupling between integrated systems (Riad et al., 2008; Hassan, 2008). For this reason, we are going to illustrate one of these scenarios in subsequent sections.

Assume that we have the following two banking systems that need to be integrated together with minimum impacts on underlying architecture and components:

- Core-bank: It is a legacy monolithic system that acts as a back-end for other operational (front-end) systems. Corebank system consists of different business modules including (customer management, deposits, foreign exchange, commercial loans, Islamic loans, etc). Additionally, this system offers some non-functional features such as transaction support, role-based security, and auditing and logging for user actions. The core-bank system allows users to send requests and receive responses via its GUI while all processing occurs into database itself. This database consists of large number of precompiled stored procedures, and each stored procedure takes a huge number of input parameters to process them and return back generated result sets.

- Loan-origination: It is a front-end system built with .NET framework 2.0 and c\# language and it is responsible for allowing bank clients to issue loans, define installments, schedule payments, etc. Because all information about bank clients and loans are stored into the core-bank system, we have to integrate our loan-origination system with it. To enable this integration, we will build an XML Web Service that comprises a large number of methods responsible for accepting requests from different client (front-end) applications including the Loan-Origination application, and passing them to stored procedures that reside under the corebanking system. To enable efficient use of the new XML Web Service, software components should be built to wrap available stored procedures and encapsulate their logic. These components will divide underlying business logic that is scattered over different stored procedures into modules that could be easily used and modified whenever needed (Sneed, 2006; Zhang, 2004). Defined components could be realized (designed and built) with any modern programming environment (such as J2EE or .NET) that supports advanced features like OOP, RAD, XML Web Services, etc. After realizing needed components, they will be placed into a new layer that resides between the new XML Web Service and under- 


\section{Journal of Computer Science \& Systems Biology - Open Access \\ JCSB/Vol.2 September-October 2009}

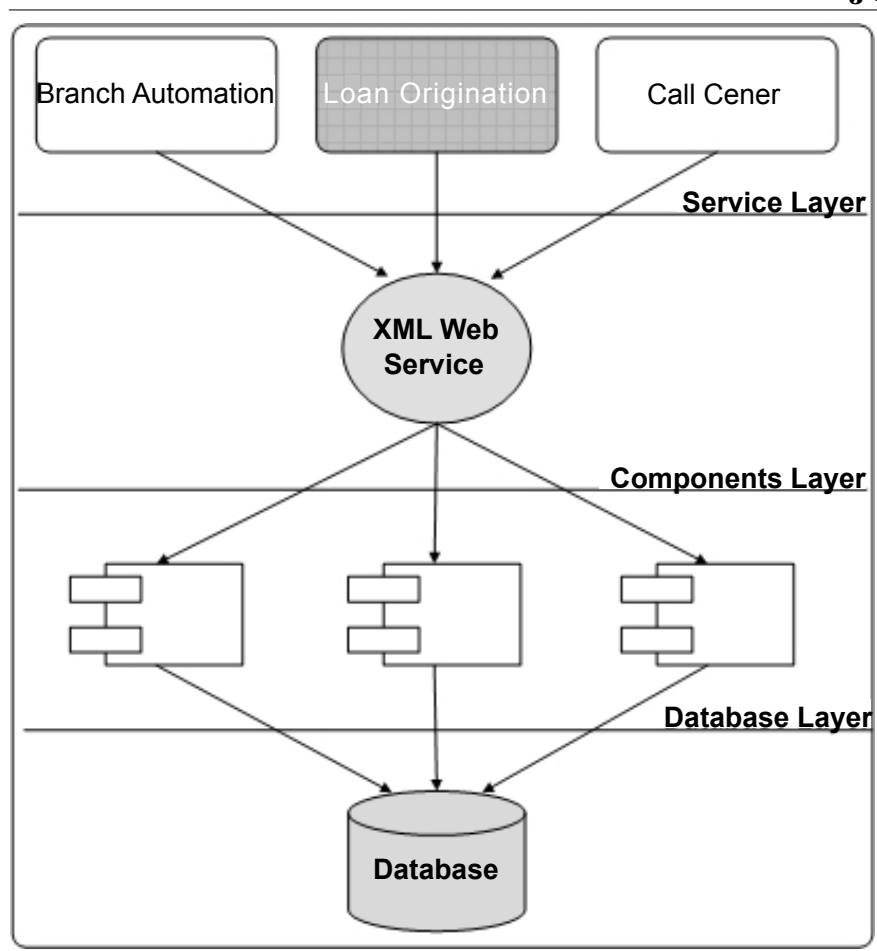

now depends on a service layer that acts as a wrapper that receives client requests and maps them to appropriate stored procedure(s). Figure 3 illustrates a comparison between the total times needed for receiving the response message of the request that gets basic information about one bank client using both methods.

\section{Optimization Tactics and Strategies}

As illustrated, the original method that uses stored procedures is 4 times faster than the new XML Web Services method. To mitigate this problem, many experiments have been conducted to yield the following list of recommended tactics and techniques:

- Utilize better encoders/decoders: Utilizing or even customizing more optimized encoders/decoders and encoding formats can save much of time needed for preparing data. UTF8 is a well known and standard encoding format that has been tuned to replace the traditional slow ASCII format. It is now known to be one of the fastest encoding options available in software market that supports almost all commonly used characters as well as special characters (Understanding Encodings).

Figure 1: Integration Architecture.

\begin{tabular}{|c|c|}
\hline Request Message & Response Message \\
\hline 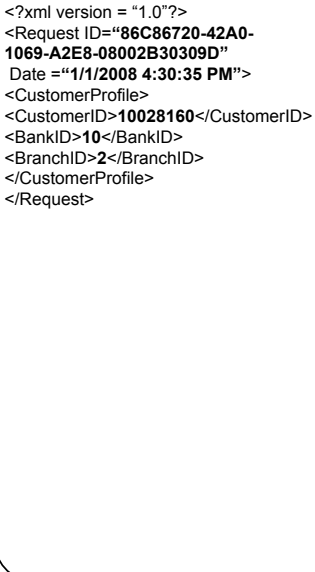 & 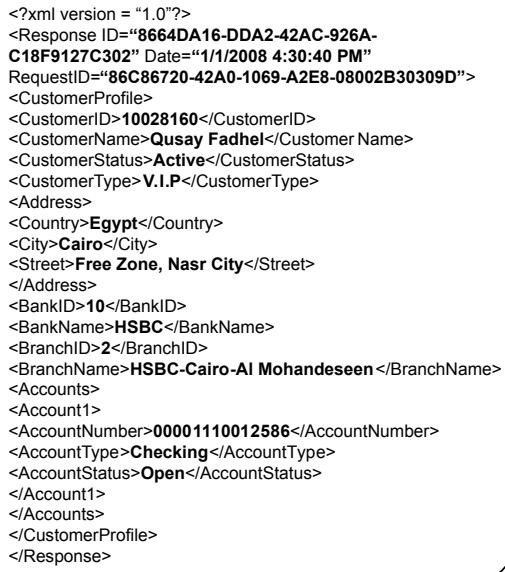 \\
\hline
\end{tabular}

Figure 2: An Example for Request/Response Messages.

lying stored procedures to act as a mediator/wrapper that accepts different invocations from XML Web Service and turn them into formats that could be accepted by stored procedures. Figure 1 illustrates a high level view for integration architecture of our systems, whereas, Figure 2 illustrates simple request and response messages that are handled by the system.

\section{Performance Evaluation}

Due to high dependency on XML Web Services, the illustrated architecture slightly suffers from low performance. In fact, leveraging SOA is always hampered by large sizes of XML files being transferred between clients and servers. These large data files always clog network and drain almost all hardware resources including RAM, CPU, and storage infrastructure. In original architecture the core-banking system was entirely built over a set of stored procedures to execute needed business logic, but it

Figure 3: Invocation Time needed for Database Stored Procedure vs. XML Web Service.

\begin{tabular}{|c|c|c|c|c|c|c|}
\hline \multicolumn{6}{|c|}{ Serialization Benchmark } & \multirow[t]{2}{*}{$x$} \\
\hline \multicolumn{2}{|c|}{ Evaluate } & \multicolumn{4}{|c|}{\begin{tabular}{|l|} 
Total Number of Columns: 5 Columns \\
Total Number of Serialized Rows: 20 Rows \\
Elapsed Time of Xml Serialization: $290 \mathrm{~ms}$ \\
Elapsed Time of Binary Serialization: $8 \mathrm{~ms}$
\end{tabular}} & \\
\hline & ID & LastName & FirstName & Courtesy & Address & $\wedge$ \\
\hline \multirow[t]{11}{*}{, } & 100100 & Davolio & Nancy & Ms. & 507 - 20th Ave. E.Apt. 2A & \\
\hline & 100101 & Fuller & Andrew & Dr. & 908 W. Capital Way & \\
\hline & 100102 & Leverling & Janet & Ms. & 722 Moss Bay Blvd. & $\equiv$ \\
\hline & 100103 & Peacock & Margaret & Mrs. & 4110 Old Redmond Rd. & \\
\hline & 100104 & Buchanan & Steven & Mr. & 14 Garrett Hill & \\
\hline & 100105 & Suyama & Michael & Mr. & Coventry HouseMiner Rd. & \\
\hline & 100106 & King & Robert & Mr. & Edgeham HollowWinchest... & \\
\hline & 100107 & Callahan & Laura & Ms. & 4726 - 11th Ave. N.E. & \\
\hline & 100108 & Dodsworth & Anne & Ms. & 7 Houndstooth Rd. & \\
\hline & 100109 & John & Fuller & Mr. & 15 Garrett Hill & \\
\hline & 100110 & Rod & Jakson & Mr. & 16 Garrett Hill & $\vee$ \\
\hline$<$ & & & & & & 8 \\
\hline
\end{tabular}

Figure 4: Performance of Binary Serialization vs. XML Serialization.

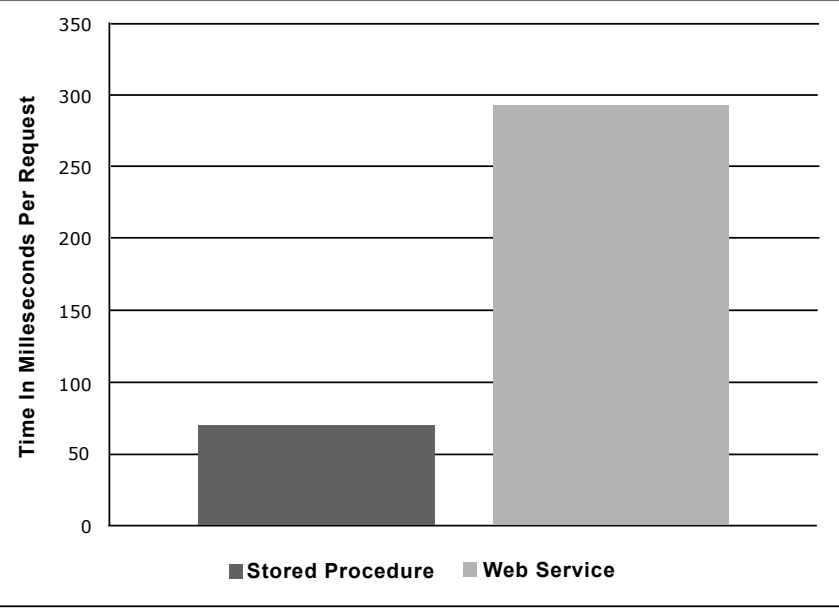




\section{Journal of Computer Science \& Systems Biology - Open Access \\ JCSB/Vol.2 September-October 2009}

- Leverage binary XML: As mentioned, most of current XML implementations depend on using plain-text format that causes data files to be very complex and large in size. W3C has announced that formatting XML data using binary format is more efficient for both network and hardware utilization (Geer, 2005). Different techniques might be utilized to use binary data, for example, the data being transmitted between network nodes might be serialized (marshaled) and de-serialized (un-marshaled) using binary format instead of text-based XML format. Figures 2, 3 illustrate the results generated by a simple benchmark (windows forms) application that was written (using c\# language and .NET Framework 3.5) to estimate the serialization time needed by binary and text-based serialization techniques on a PC that uses Intel Dual Core 2.6 GHz processor and $2 \mathrm{~GB}$ memory. As depicted, the application shows that XML serialization takes 290 milliseconds to serialize some of stored (dummy) information. The amount of data is represented in 20 rows and 5 columns ( 948 bytes), whereas, the binary serialization takes only 8 milliseconds to serialize the same data. This means that binary serialization in our scenario saves more than $97 \%$ of time needed by XML serialization. Certainly the serialization time will vary according to number of factors including amount and complexity of data being serialized, for instance, if we serialized 10,000 rows of the aforementioned data $(569,808$ bytes $)$ on the same PC, the XML serialization will take 709 milliseconds versus 322 milliseconds for binary serialization, which means that binary serialization saves more than $55 \%$ of total serialization time. We should note that there is a fixed time that is needed in each method whatever the size and amount of data to initialize serialization process. This initialization operation is solely needed to read the schema of data being serialized. Figure 5 illustrates a simple comparison between total times needed for binary serialization versus XML (text-based) serialization to return the response of a simple request.

- Apply data compression techniques: Since XML is a textbased format, and XML documents always have too many white spaces, then using traditional compression algorithms such as ZIP/GZIP for compressing data transferring between clients and servers can get rid of many bytes of the volume out of data files. To apply compression technique on travel-

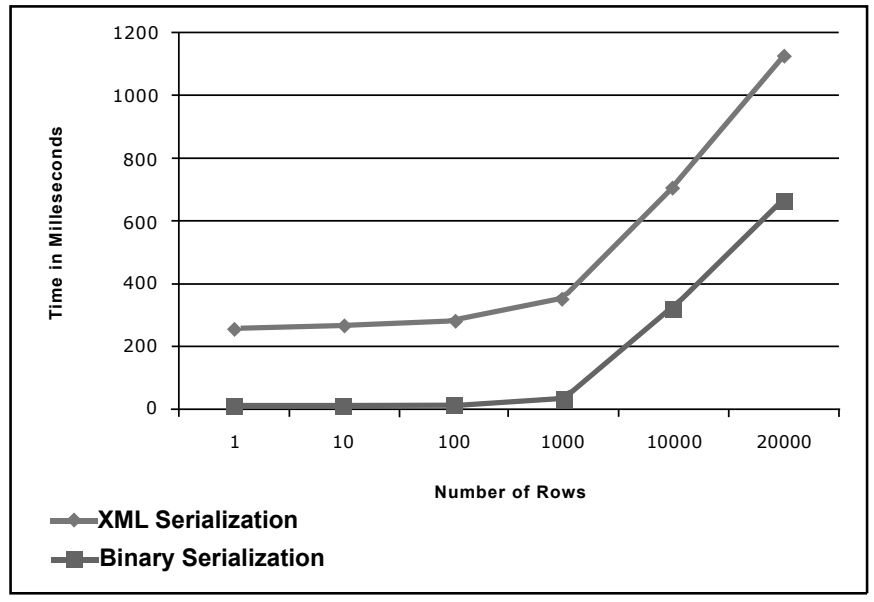

Figure 5: Binary Serialization vs. XML Serialization.

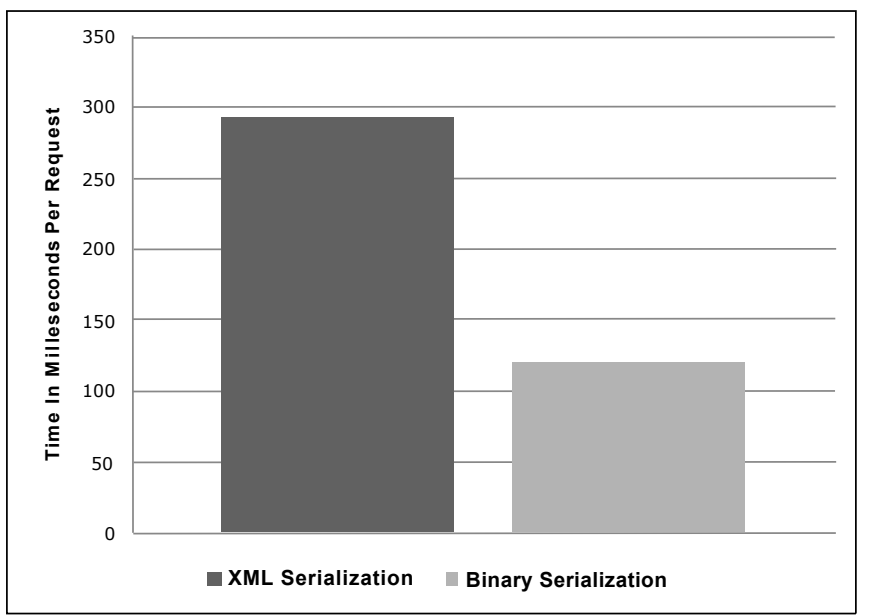

Figure 6: Invocation Time needed for XML Serialization vs. Binary Serialization.

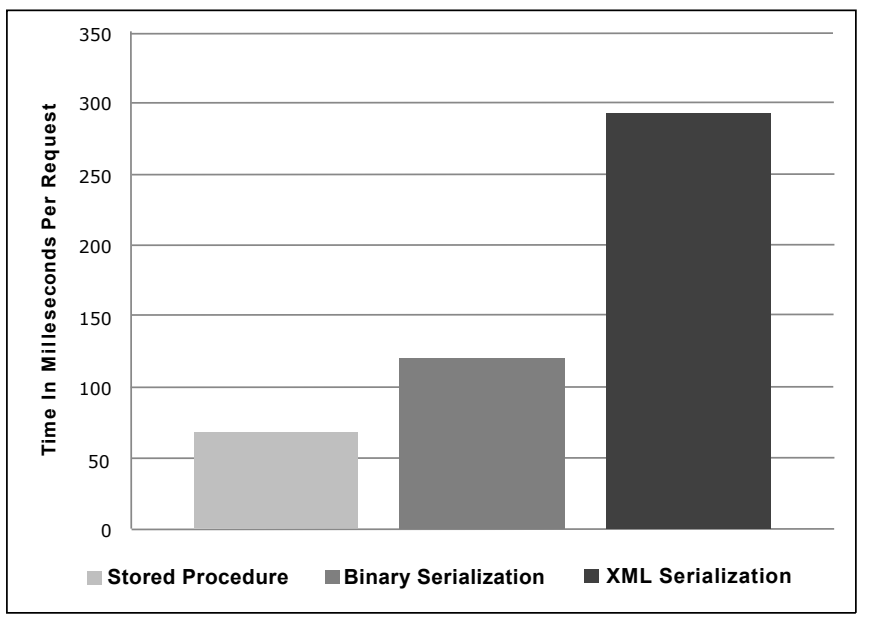

Figure 7: Invocation Time needed for Stored Procedures vs. Binary Serialization vs. XML Serialization.

ing data, both requestors and responders must understand the used compression algorithm to be able to recognize and use these data. This assurance should be identified and guaranteed by SOA governance team during preliminary implementation phases. Figure 8 illustrates the results generated by a benchmark (console) application that has been written (using c\# language and .NET Framework 3.5) to calculate total save in size of Response document that was illustrated in figure 2 using ZIP/GZIP algorithms. The results shows that the original size of Response document was 771 bytes, whereas, the size of compressed file is only 550 bytes which means that ZIP/GZIP algorithms save 221 bytes (approximately 29\%) from original document size. Another way that could make XML documents smaller is to avoid long element and attribute names. For example, the Response document illustrated in figure 2 could be abbreviated as illustrated in figure 9. The size of new abbreviated Response document takes only 559 bytes with total save 212 bytes (approximately 28\%) from original document size. This option will only be applicable where the human readability of request/response messages is not required. Combining two techniques together in our scenario allowed us to eliminate about $57 \%$ (the most) of total document size which is excellent to our issue while keeping the great benefits of SOA 


\section{Journal of Computer Science \& Systems Biology - Open Access \\ JCSB/Vol.2 September-October 2009}

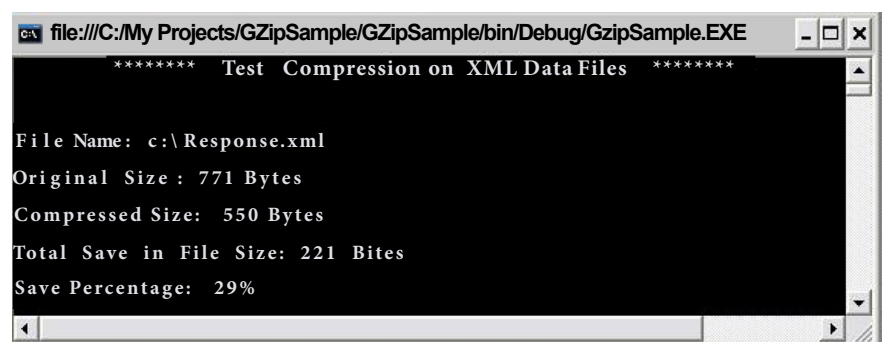

Figure 8: Total Save in Response Document Using ZIP/GZIP Algorithms.

\begin{tabular}{|c|c|}
\hline Request Message & Response Message \\
\hline 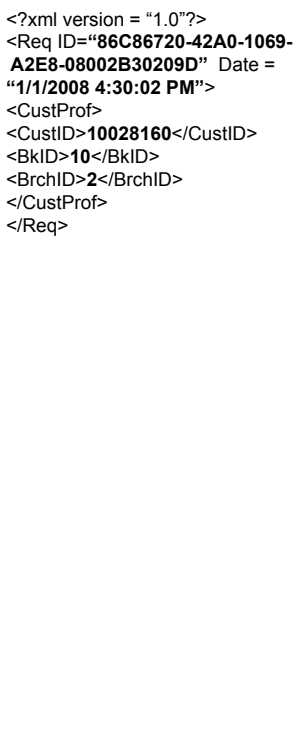 & 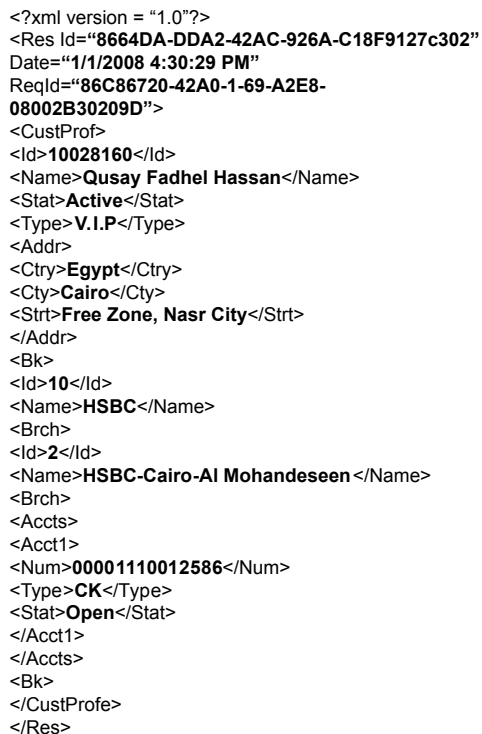 \\
\hline
\end{tabular}

Figure 9: Abbreviated Request/Response Messages.

including the ease-of-use and simplicity of XML Web Services and overall architecture. Certainly, these values may vary depending on the structure and size of original documents being compressed, however, saving total size of documents (especially complex and large ones) being processed and transmitted over network has with no doubt positive impact on memory consumption, network utilization, and transmission time. One note that we should take into consideration regarding to applying ZIP/GZIP algorithms is that processing compressed data may require more CPU usage at the receiving (last) node, as it will be a part of its responsibilities to unzip data in order to be able to use it in any further processing. This issue can be easily resolved by using more powerful servers that use high speed multi-processing cores.

- Use better parsers: SAX and DOM are the most popular parsing techniques available in XML market. Many benchmarks on their throughputs have shown that they require slightly long times to parse XML files at different sizes and complexity levels. The long time is mainly needed because these parsers read data files more than once (at least two times) to be able to discover their structure and to validate entire data. Furthermore, big amount of memory might be needed to store extracted data during parsing phase, and this of course may degrade overall performance of used CPUs when no more memory is available (in paging and caching operations performed by operating system). To resolve this issue, we may depend on faster and more efficient techniques such as Virtual Token Descriptor XML (VTD-XML) which depends on "non-extractive" tokenization approach to parse XML files (VTD-XML: The Future of XML Processing). Additionally, using schema-specific parsers rather than general purpose parsers can greatly enhance the performance of parsing phase (Chiu et al., 2002). Using more enhanced parsing algorithms and tools can save time needed to parse data which in turn save the overall processing time and resources.

- Pre-generate serialization assemblies: Many of development tools (including .NET 2.0 and latter) allow developers to pre-generate and cache serialization assemblies that could be deployed with applications to save time needed for discovering, extracting, and recognizing structures of objects being serialized and de-serialized (XML Serializer Generator Tool' (Sgen.exe)).

- Divide large files: It is known that large files always have bad impacts on the utilization of hardware resources (memory, processor, and network) and processing time. Dividing large and complex data files into smaller pieces (if possible) can deliberately enhance both processing and network performance.

- Install silicon-based XML engines: Many silicon-based engines are available now to handle XML at higher speeds. These engines can be embedded into different network and hardware equipments including switches, routers, load balancers, PCI-cards and servers (WebSphere DataPower SOA Appliances).

- Apply parallelism techniques: Data files could be yielded and processed in parallel using grid-based technologies that depend on mutli-threaded systems (one thread for each process/sub-process) to allow faster processing and better utilization of available hardware resources including processing cycles, memory, and storage infrastructure (Pan et al., 2007; Lu et al., 2008; Cameron et al., 2008).

- Utilize high speed networks: There are now Ethernet implementations that enable enterprises to have transmission speed that varies from traditional cdfdc x10Mbits/s Ethernet to 100Gigabit Ethernet (http://www.ieee802.org/3/ba/PAR/ par_0308.pdf; http://www.ieee802.org/3/hssg/; http:// www.ieee802.org/3/ba/public/index.html; Ambrosia, 2009). Also, fiber channels and links could be installed to allow high speed and reliable transmission for data encapsulated into XML requests and replies.

\section{Conclusion}

This paper has presented an evaluation for XML Web Services in real-time business systems by illustrating a common scenario for two banking systems that need to be integrated together with contemporary SOA methodologies and terms. The use of XML Web Services in our scenario caused a number of problems including slow performance and bad utilization for hardware and network recourses over RPC implementation (stored procedures) that is widely used in traditional point-topoint integration methods. These pitfalls could not be accepted under any circumstances in real-time business systems that re- 


\section{Journal of Computer Science \& Systems Biology - Open Access \\ JCSB/Vol.2 September-October 2009}

ceive and handle tons of requests every single second. For that reason, the paper has given some tips and recommended actions that could be taken by implementers to allow efficient and better use of SOA and XML Web Services in building and integrating real-time business applications and systems.

\section{References}

1. Ambrosia JD (2009) IEEE P802.3ba Objectives.

2. Bustamante FE, Eisenhauer G, Schwan K, Widener P(2000) Efficient wire formats for high performance computing. Proceedings of the 2000 Conference on Supercomputing. »CrossRef » Google Scholar

3. Cameron RD, Herdy KS, Lin D (2008) High Performance XML Parsing Using Parallel Bit Stream Technology. http:// www.cs.sfu.ca/ cameron/parabix-study-preprint.pdf "CrossRef » Google Scholar

4. Channabasavaiah K, Holley K, Tuggle E (2003) Migrating to a service-oriented architecture. Part 1, 2. http://www128.ibm.com/developerworks/library/ws-migratesoa "CrossRef » Google Scholar

5. Chiu K, Govindaraju M, Bramley R (2002) Investigating the limits of SOAP performance for scientific computing. Proceedings of the 11th IEEE International Symposium on High Performance Distributed Computing pp246-254. »CrossRef » Google Scholar

6. Curbera F, Duftler M, Khalaf R, Nagy W, Mukhi N, et al. (2002) Unraveling the web services web: An introduction to SOAP, WSDL, UDDI. IEEE Internet Computing 6: 86-93. »CrossRef » Google Scholar

7. Extensible Markup Language (XML) Specification Version 1.0 (2004) W3C. http://www.w3.org/tr/rec-Xml/

8. Fielding R, Gettys J, Mogul J, Frystyk H, Masinter L, et al. (1999) Hypertext transfer protocol - HTTP/1.1, 1999. IETF RFC 2616. http://www.ietf.org/rfc/rfc2616.txt »CrossRef " Google Scholar

9. Geer D (2005) Will Binary XML Speed Network Traffic?. IEEE Computer Society. » CrossRef » Google Scholar

10. Hassan QF (2008) Important Aspects of SOA: A Survey. The 2nd International Conference on Business and Information Technologies. New approaches 24-25.

11. http://www.ieee802.org/3/ba/PAR/par_0308.pdf
12. http://www.ieee802.org/3/ba/public/index.html

13. http://www.ieee802.org/3/hssg/

14. Krafzig D, Banke K, Slama D, (2005) Enterprise SOA Service-Oriented Architecture Best Practices. Prentice Hall.

15. Lu W, Chiu K, Pan Y A (2008) Parallel Approach to XML Parsing. http://grid.cs.binghamton.edu/projects/publications/ parallel-Grid06/parallel-Grid06.pdf "CrossRef » Google Scholar

16. Pan Y, Zhang Y, Chiu K, Lu W (2007) Parallel XML Parsing Using Meta-DFAs. Third IEEE International Conference on e-Science and Grid Computing (e-Science 2007) pp237-244. »CrossRef » Google Scholar

17. Papazoglou MP (2003) Service-Oriented Computing: Concepts, Characteristics, and Directions", Proceedings of the fourth IEEE international conference on web information systems engineering (WISE'03). »CrossRef » Google Scholar

18. Riad AM, Hassan A, Hassan QF (2008) Leveraging SOA in Banking Systems' Integration. Journal of Applied Economics Science 2: 145-157. »CrossRef » Google Scholar

19. Sneed HM (2006) Integrating legacy Software into a Service oriented Architecture. Proceedings of the Conference on Software Maintenance and Reengineering (CSMR'06), IEEE. »CrossRef » Google Scholar

20. Understanding Encodings. http://msdn.microsoft.com/en-us/ library/ms404377.aspx

21. VTD-XML: The Future of XML Processing. http://vtdxml.sourceforge.net/

22. WebSphere DataPower SOA Appliances. http://www01.ibm.com/software/integration/datapower/

23. XML Serializer Generator Tool' (Sgen.exe). http:// msdn.microsoft.com/en-us/library/bk3w6240.aspx

24. Zhang Z, Yang H (2004) Incubating Services in Legacy Systems for Architectural Migration. Proceedings of the 11th Asia-Pacific Software Engineering Conference (APSEC'04), IEEE. »CrossRef » Google Scholar 